\title{
DOUBLE COMMUTANTS OF OPERATORS QUASI-SIMILAR TO NORMAL OPERATORS
}

\section{KATSUTOSHI TAKAHASHI}

ABSTRACT. It is shown that double commutants of operators quasi-similar to normal operators are reflexive.

An algebra $\mathscr{A}$ of bounded linear operators on a Hilbert space is said to be reflexive if $\mathscr{A}=$ Alg Lat $\mathscr{A}$, where Lat $\mathscr{A}$ denotes the family of all subspaces invariant under all elements of $\mathscr{A}$ and Alg Lat $\mathscr{A}$ is the algebra of all operators $X$ for which $X \mathscr{M} \subseteq$ $\mathscr{M}$ for every $\mathscr{M} \in$ Lat $\mathscr{A}$. If $\mathscr{A}$ is a von Neumann algebra, then $\mathscr{M} \in$ Lat $\mathscr{A}$ means that the projection to $\mathscr{M}$ belongs to the commutant $\mathscr{A}^{\prime}$, therefore Alg Lat $\mathscr{A}$ coincides with the double commutant $\mathscr{A}^{\prime \prime}$. Thus the von Neumann double commutant theorem can be rephrased as follows (cf. [4, Theorem 9.17]): every von Neumann algebra is reflexive.

Recall some definitions. For operators $T_{1}$ and $T_{2}$, let us write $T_{1} \prec T_{2}$ (resp. $T_{1} \prec T_{2}$ ) to denote that there exists a quasi-affinity, i.e. injection with dense range (resp. an injection) $X$ such that $X T_{1}=T_{2} X . T_{1}$ and $T_{2}$ are quasi-similar by definition if $T_{1} \prec T_{2}$ and $T_{2} \prec T_{1}$.

If $N$ is a normal operator, the commutant $\{N\}^{\prime}$ becomes a von Neumann algebra by the Fuglede theorem, so that both the commutant and the double commutant $\{N\}^{\prime \prime}$ are reflexive. If an operator $T$ is quasi-similar to a normal operator $N$, the commutant $\{T\}^{\prime}$ is reflexive because the reflexivity of commutant is preserved under quasi-similarity [1] and $\{N\}^{\prime}$ is reflexive. In this note we shall show that the double commutant $\{T\}^{\prime \prime}$ is reflexive too.

THEOREM. If an operator $T$ is quasi-similar to a normal operator $N$, then the double commutant $\{T\}^{\prime \prime}$ is reflexive.

Before going into the proof, let us present an immediate consequence.

COROllary. If $T$ is a contraction of class $C_{11}$, that is, $\lim _{n \rightarrow \infty}\left\|T^{n} x\right\| \neq 0$ and $\lim _{n \rightarrow \infty}\left\|T^{* n} x\right\| \neq 0$ for every nonzero $x$, then the double commutant $\{T\}^{\prime \prime}$ is reflexive.

In fact, it is well known that a contraction of class $C_{11}$ is quasi-similar to a unitary operator (see [5, Proposition II.3.5]).

Received by the editors November 11, 1983 .

1980 Mathematics Subject Classification. Primary 47C05, 47B15; Secondary 47A45.

Key words and phrases. Double commutant, reflexive, quasi-similar, normal operator, $C_{11}$-contraction. 
This result for a $C_{11}$-contraction $T$ was proved in [3, Theorem 3] under the condition that every injection in $\{T\}^{\prime}$ has dense range.

For the proof of the theorem we need a lemma, which is an analog of [3, Lemma 5] for $C_{11}$-contractions.

LEMma. Let $T$ be an operator quasi-similar to a normal operator. Then, for $\mathscr{M} \in \mathrm{Lat} T$ (= the family of all $T$-invariant subspaces), the following conditions are mutually equivalent.

(i) $T \mid \mathscr{M}$ is quasi-similar to a normal operator.

(ii) $T \mid \mathscr{M}>N_{1}$ for some normal operator $N_{1}$.

(iii) $\mathscr{M}=(\operatorname{ran} Z)^{-}$for some $Z \in\{T\}^{\prime}$.

If one of these conditions holds, then $\mathscr{M} \in \operatorname{Lat}\{T\}^{\prime \prime}$.

Proof. Let $X$ and $Y$ be quasi-affinities such that $X T=N X$ and $T Y=Y N$ for some normal operator $N$ on $\mathscr{G}$. (i) $\Rightarrow$ (ii) is trivial.

(ii) $\Rightarrow$ (iii): Since $N_{1} \prec T|\mathscr{M} \prec N|(X \mathscr{M})^{-}$, it follows from [2, Lemma 4.1] that $(X \mathscr{M})^{-}$is $N$-reducing and $N \mid(X \mathscr{M})^{-}$is unitarily equivalent to $N_{1}$. Therefore we have $(T \mid \mathscr{M})^{*} \prec N_{1}^{*} \prec N^{*} \mid(X \mathscr{M})^{-} \stackrel{i}{\prec} N^{*} \prec T^{*}$. Denote by $W$ the injection from $\mathscr{M}$ to $\mathscr{H}\left(=\right.$ the space on which $T$ acts) such that $W(T \mid \mathscr{M})^{*}=T^{*} W$, and by $J$ the inclusion mapping of $\mathscr{M}$ into $\mathscr{H}$. Then $Z=J W^{*}$ is an operator required in (iii).

(iii) $\Rightarrow$ (i): Define an operator $K$ from $\mathscr{G}$ to $\mathscr{M}$ by $K x=Z Y x$ for $x \in \mathscr{G}$. Then $K$ has dense range and $(T \mid \mathscr{M}) K=K N$. Also $(X \mid \mathscr{M})(T \mid \mathscr{M})=N(X \mid \mathscr{M})$ and $X \mid \mathscr{M}$ is injective. Therefore it follows from [2, Lemma 4.1] that $N \mid(\operatorname{ker} K)^{\perp}$ and $N \mid(X \mathscr{M})^{-}$ are unitarily equivalent normal operators, and so $T \mid \mathscr{M}$ is quasi-similar to a normal operator $N \mid(X \mathscr{M})^{-}$. Finally it is clear that $\mathscr{M} \in \operatorname{Lat}\{T\}^{\prime \prime}$ for any subspace $\mathscr{M}$ satisfying (iii).

Proof OF THEOREM. By assumption there are quasi-affinities $X$ and $Y$ satisfying $X T=N X$ and $T Y=Y N$. Without loss of generality, the product $X Y$ can be assumed to be nonnegative. Indeed, since $X Y \in\{N\}^{\prime}, X Y$ admits the polar factorization $X Y=U P$ in the von Neumann algebra $\{N\}^{\prime}$, where $U$ is unitary and $P$ is nonnegative. The quasi-affinities $U^{*} X$ and $Y$ satisfy the required condition.

The inclusion $\{T\}^{\prime \prime} \subseteq \operatorname{Alg} \operatorname{Lat}\{T\}^{\prime \prime}$ is obvious. To see the converse inclusion, take $A \in \operatorname{Alg} \operatorname{Lat}\{T\}^{\prime \prime}$. In order to show $A \in\{T\}^{\prime \prime}$, it suffices to prove that $A$ commutes with $Y C X$ for all $C \in\{N\}^{\prime}$. In fact, then $A$ commutes with $Y X$, and also with $Y X B Y X$ for any $B \in\{T\}^{\prime}$, because $X B Y$ belongs to $\{N\}^{\prime}$. Therefore we have

$$
Y X B A Y X=Y X B Y X A=A Y X B Y X=Y X A B Y X .
$$

Then the quasi-affinity properties of $X$ and $Y$ imply $B A=A B$, hence $A \in\{T\}^{\prime \prime}$.

Let us show that $A$ commutes with $Y C X$ for all $C \in\{N\}^{\prime}$. Since $\{N\}^{\prime}$ is a von Neumann algebra, we have only to show that $A(Y H X)=(Y H X) A$ for any selfadjoint injection $H \in\{N\}^{\prime}$. Take $\mathscr{M} \in \operatorname{Lat}\{X Y H X Y\}^{\prime}$. Since $\{N\}^{\prime \prime} \subseteq\{X Y H X Y\}^{\prime}$, we have $\mathscr{M} \in \operatorname{Lat}\{N\}^{\prime \prime}$, hence $\mathscr{M}$ is $N$-reducing and $N \mid \mathscr{M}$ is normal. Then, since $(Y \mathscr{M})^{-} \in$ Lat $T$ and $T\left|(Y \mathscr{M})^{-} \succ N\right| \mathscr{M}$, it follows from the Lemma that $(Y \mathscr{M})^{-} \in$ Lat $\{T\}^{\prime \prime} \subseteq$ Lat $A$, and so $X Y H X A Y \mathscr{M} \subseteq(X Y H X Y \mathscr{M})^{-} \subseteq \mathscr{M}$. We can conclude that $X Y H X A Y \in \operatorname{Alg} \operatorname{Lat}\{X Y H X Y\}^{\prime}$. Then, since the commutant $\{X Y H X Y\}^{\prime}$ of the 
selfadjoint operator $X Y H X Y$ is reflexive, $X Y H X A Y \in\{X Y H X Y\}^{\prime}$. Taking $H=I$, we have $(X Y)^{2} X Y X A Y=X Y X A Y(X Y)^{2}$ and $(X Y)^{2} X A Y=X A Y(X Y)^{2}$ by the injectivity of $X Y$. Since $X Y$ is assumed to be nonnegative, taking the square root of $(X Y)^{2}$, we obtain $X Y X A Y=X A Y X Y$. Then for any selfadjoint injection $H$ we have

$$
X Y H X Y X Y H X A Y=X Y H X A Y X Y H X Y=X Y H X Y X A Y H X Y \text {. }
$$

Finally it follows from the injectivity of $X Y H X Y X$ and the dense range property of $Y$ that $Y H X A=A Y H X$.

\section{REFERENCES}

1. H. Bercovici, C. Foiaş and B. Sz.-Nagy, Reflexive and hyper-reflexive operators of class $C_{0}$, Acta Sci. Math. 43 (1981), 5-13.

2. R. G. Douglas, On the operator equation $S^{*} X T=X$ and related topics, Acta Sci. Math. 30 (1969), $19-32$.

3. L. Kerchy, On invariant subspace lattices of $C_{11}$-contractions, Acta Sci. Math. 43 (1981), 281-293.

4. H. Radjavi and P. Rosenthal, Invariant subspaces, Springer-Verlag, Berlin, Heidelberg and New York, 1973.

5. B. Sz.-Nagy and C. Foiaş, Harmonic analysis of operators on Hilbert space, North-Holland, Amsterdam, 1970.

Department of Mathematics, Sapporo Medical College, Minami-1 Nishi-17, Sapporo, Japan 